\title{
A Política Comparada na América \\ Latina: dilemas e desafios no Brasil
}

Comparative Politics in Latin America:

\section{Rodrigo Stumpf González Marcello Baquero}

\section{Resumo}

Este artigo busca analisar a evolução dos estudos de Política Comparada sobre a América Latina, com ênfase especial sobre o caso brasileiro. Inicialmente é apresentada a compreensão conceitual do que pode ser enquadrado como estudo comparativo na Ciência Política. Para avaliar a situação atual da pesquisa comparativa no continente e no Brasil, primeiramente, foi examinado o volume da produção recente mediante a análise dos artigos publicados em algumas das revistas mais prestigiadas sobre o tema, no âmbito do continente, e nas principais revistas de ciência política no Brasil. A partir da constatação do pequeno peso dos estudos comparativos sobre o total da produção examinada, busca-se discutir a forma como o método comparativo tem sido abordado nos programas de pós-graduação em Ciência Política, seja do ponto de vista da formação, seja na dimensão metodológica em teses e dissertações. Por fim, são debatidas medidas possíveis no sentido de fomentar os estudos comparativos no futuro.

\section{Palavras-chave}

Política Comparada; América Latina; Brasil.

\begin{abstract}
This article seeks to analyze the evolution of Comparative Political studies about Latin America, highlighting the Brazilian case. The paper begins with a conceptual understanding of what could be considered as comparative studies in Political Science. In order to evaluate the present situation of comparative research in this region, and especially in Brazil, the scientific production was examined giving emphasis to published articles in the most prestigious journals of political science in Brazil. As it was verified the small weight of comparative studies over the total studies of political science, the study attempts to discuss how the comparative method has been employed in political science graduate programs, whether from the point of view of human resources formation as well as in the methodological dimension as it appeared in thesis and dissertations. Finally we debate possible measures that could foment comparative studies in the future.
\end{abstract}

\section{Keywords}

Comparative Politics; Latin America; Brazil. 


\section{Introdução}

A área de política comparada na América Latina tem atravessado por diversas fases, tanto do ponto de vista teórico quanto metodológico. Os estudos tradicionais de política comparada que enfatizavam comparações ao nível macro (nações), foram deslocados por abordagens que valorizavam a operacionalização de variáveis "universais" com base na técnica de comparação por semelhanças ou diferenças (PRZEWORSKI, 1970). No entanto, tais esforços não foram produtivos, sinalizando a necessidade de comparar sem desconhecer a importância do contexto histórico estrutural. Nesse sentido, nos encontramos numa situação onde os velhos dilemas produzidos pela comparação macro-histórica, não solucionados, retornam como respostas no contexto atual de valorização excessivamente empírica. Torna-se, portanto, relevante analisar como a política comparada está sendo utilizada na região latino-americana.

Nesse cenário, a América Latina, como definição geográfica de um subcontinente, embora possa sofrer contestações do ponto de vista da definição de seus limites ${ }^{1}$, é, inegavelmente, importante do ponto de vista político e acadêmico. Assim são múltiplos os centros de estudo, publicações, associações acadêmicas e congressos organizados sobre este tema, podendo destacar-se associações e congressos como os da LASA - Latin American Studies Association, da ALACIP - Associação Latinoamericana de Ciencia Política, da ALAS - Associación Latinoamericana de Sociologia, do CEISAL - Consejo Europeu de Investigaciones en America Latina. Além das revistas como Latin American Research Review, publicada pela LASA, América Latina Hoy, publicada pela Universidade de Salamanca, Latin American Politics and Society, publicada pela Universidade de Miami e ainda a América Latina, publicada (em russo) pelo Instituto de América Latina da Academia de Ciências da Russia, entre tantas outras.

Por outro lado, há indicativos de que a área de Ciência Política tem crescido e se institucionalizado na região nas últimas duas décadas, provavelmente beneficiada pelo mais longo período de estabilidade (ainda que relativa) democrática na Região. Em 2012, foi criada a Associação Iberoamericana de Associações de Ciência Política, que reúne, além dos parceiros da península Ibérica, associações nacionais de Argentina, Brasil, Chile, Colômbia, Equador, México e Uruguai. Cada uma destas associações tem realizado congressos nacionais com a participação de significante número de expositores, não apenas nacionais, mas abrangendo de outros países. Cabe destacar que, no ano

\footnotetext{
1 O pertencimento ou não à América Latina costuma ser definido pela origem linguística dos colonizadores europeus, em especial os ibéricos. Em um período mais recente, o termo "latino" tem sido utilizado nos Estados Unidos da América para identificar populações imigrantes oriundas, principalmente, da América Central, México e Caribe, deixando de incluir, por exemplo, a comunidade ítalo-americana, que seria, em última instância, originariamente latina.
} 
de 2013, a ALACIP realizou seu sétimo congresso, com a participação de associados dos diferentes países da região.

Desse modo, as condições materiais e institucionais para a circulação de pesquisadores e o estabelecimento de redes de pesquisa melhoraram muito nos últimos anos, com uma contribuição não desprezível do desenvolvimento das tecnologias de comunicação neste processo.

Até que ponto estes processo se refletiram no fortalecimento de estudos comparativos na ciência política, tendo por foco a América Latina? E no Brasil, qual espaço tem sido dado no mundo acadêmico para a política comparada? Este tópico do trabalho busca avançar no debate desta questão.

Isto nos remete para um problema inicial, que é definir do que estamos falando, ao referir-se à política comparada. A partir desta definição, este texto busca traçar um panorama do estudo da política comparada no Brasil no período recente.

\section{Comparação: método ou conteúdo}

A Ciência Política é uma das "ciências" mais problemáticas para desenvolver teoremas universais, em virtude não somente da dificuldade inerente a própria temática, mas pela variedade teórico-metodológica que utiliza.

Decorrente dessa diversidade há uma riqueza de temas a serem abordados pela Ciência Política, na perspectiva da Política Comparada. No entanto, pela própria dinâmica desta área, nos seus vários níveis (micro, meso e macro), o que é verdade hoje pode mudar radicalmente amanhã. Ao contrário das ciências exatas, que realizam suas pesquisas dentro de laboratórios, os cientistas políticos precisam trabalhar com temas e fatos que mudam constantemente. Tal situação torna difícil o estabelecimento de leis sociológicas universais que se sustentem no tempo e no espaço.

Presentemente, existe uma variedade ampla de "abordagens" consideradas adequadas para analisar a política e sua dinâmica subjacente. Tal situação tem produzido a ausência de um consenso a respeito da forma mais adequada de examinar os temas relacionados do ponto de vista comparativo. Lane já sinalizava que "presentemente o único consenso parece ser de que nada de que tem sido testado, tem funcionado e nada que possa funcionar ainda não tem sido testado" (1997, p. 4). Assim, ao contrário da sociologia que identifica os precursores da área, tais como Comte ou Durkheim, a Ciência Política parece carecer de tais precursores ou, no outro extremo, tem muitos precursores.

No caso da política comparada podemos identificar diferentes formas de tratar o tema, tendo as principais correntes as de:

- Samuel Huntington (1968): sobre política institucional;

- Przeworski e Sprague (1986): sobre socialismo eleitoral;

- Robert Dahl (1966): sobre oposições políticas;

- North e Thomas (1973): sobre desenvolvimento político econômico; 
- Barrington Morre (1966): sobre práticas para a modernidade;

- Joel Migdal (1988): sobre construção do Estado.

\section{O que significa comparar?}

Longe vai o tempo no qual Durkheim (1987) afirmou que comparar fazia parte da essência da ciência social. Na segunda metade do século XX muito foi escrito sobre a natureza da política comparada, com forte ênfase no método comparativo.

Se a política comparada é definida pela dimensão metodológica se poderia considerar como comparativo qualquer estudo que busque explicações para os fenômenos políticos a partir da comparação de unidades de análises escolhidas, para contrastar suas semelhanças ou diferenças, podendo estas unidades serem instituições políticas locais, regionais, nacionais, supra-nacionais ou outros construtos teóricos obtidos via surveys como atitudes e/ou comportamentos de indivíduos.

No entanto, há um subgrupo dentro desta abordagem que tem relevância especial no âmbito deste debate - somente aquele que toma como unidades de análise primária a totalidade das unidades políticas sob a forma de países e realizações entre duas ou mais destas unidades.

Há que se diferenciar, no entanto, esta forma de praticar a política comparada da simples justaposição, em uma mesma publicação, de estudos descritivos a respeito de cada uma das unidades nacionais, tradição criticada pelos teóricos da metodologia comparativa nos anos 1950 e 1960 do século XX (MACRIDIS e BROWN, 1968), mas ainda largamente presente na produção da área. Podem ser citados como exemplos os textos clássicos das décadas de 1980 e 1990 sobre as transições democráticas da América Latina e Europa, nos quais o conteúdo comparativo em geral se restringia a um capítulo - ou de conclusões -, escrito pelos organizadores.

O sentido estrito de política comparada que este texto propõe utilizar para análise combina os dois aspectos acima referidos: a comparação macro e microssistemica.

Não significa que estudos comparativos de outras unidades de análise, como uma comparação entre comportamento de bancadas partidárias ou governos regionais (nível intermediário) ou estudos de caso, de caráter histórico, não sejam relevantes e tenham seu espaço dentro da ciência política. Porém o espaço para sua discussão é outro, sendo perfeitamente atendido, em geral, pelas atividades das diferentes associações locais de profissionais da ciência política de nossos países.

Por outro lado, os estudos comparativos transnacionais têm um papel importante no entendimento de nossos problemas comuns e nas formas de enfrentá-los. A contribuição especial que uma rede de investigadores pode dar é 
fomentar o intercâmbio de dados que favoreçam a ampliação de estudos comparativos que levem em conta nossos países.

\section{Comparação e América Latina}

Para tentar avaliar o espaço que estudos comparativos, enfocando a América Latina, têm obtido, foi avaliada a produção dos últimos cinco anos de algumas revistas científicas especializadas em estudos comparativos, revistas especializadas em América Latina e revistas da área de Ciência Política, consideradas como referência no continente.

Embora não deva ser descartada a importância de produções sob a forma de livros ou coletâneas de múltiplos autores, na atualidade a produção em revistas acadêmicas, em seu formato eletrônico, tem sido o veículo mais efetivo de difusão do conhecimento, podendo ser consideradas, se não um indicador suficiente do que tem sido produzido, pelo menos um indicador eventual do prestígio e volume de produção em determinada temática.

Nos artigos encontrados nestas revistas o termo América Latina é utilizado em diferentes acepções, incluindo:

a) Comparação por semelhança: os artigos que comparam longitudinalmente a maioria dos países da região, com base em bancos de dados, selecionados pelo seu pertencimento ao subcontinente;

b) Comparação pela diferença: artigos que comparam um pequeno número de países da região, utilizando o contraste como motivação para escolha dos casos;

c) Estudo de caso com identificação geográfica: artigos em que o termo América Latina é utilizado apenas como referência geográfica do país analisado.

No Quadro 1 são apresentadas as características gerais das revistas analisadas. 
Quadro 1 - Revistas Acadêmicas e Foco de Análise

\begin{tabular}{|c|c|c|c|c|c|}
\hline \multirow{2}{*}{ Revista } & $\begin{array}{l}\text { Números } \\
\text { por ano }\end{array}$ & & Foco & & Língua \\
\hline & & $\begin{array}{c}\text { América } \\
\text { Latina }\end{array}$ & $\begin{array}{c}\text { Estudos } \\
\text { Comparativos }\end{array}$ & $\begin{array}{l}\text { Ciência } \\
\text { Política }\end{array}$ & \\
\hline $\begin{array}{c}\text { Comparative } \\
\text { Political Studies }\end{array}$ & 12 & & $\mathrm{X}$ & $\mathrm{X}$ & Inglês \\
\hline Comparative Politics & 4 & & $\mathrm{X}$ & $\mathrm{X}$ & Inglês \\
\hline América Latina Hoy & 3 & $\mathrm{X}$ & & $\mathrm{X}$ & Espanhol \\
\hline $\begin{array}{l}\text { Journal fo } \\
\text { Democracy }\end{array}$ & 4 & & & $\mathrm{X}$ & Inglês \\
\hline $\begin{array}{l}\text { Revista de Ciencia } \\
\text { Política (Chile) }\end{array}$ & 3 & & & $X$ & $\begin{array}{l}\text { Espanhol/ } \\
\text { Inglês }\end{array}$ \\
\hline $\begin{array}{c}\text { Latin American } \\
\text { Politics and Society }\end{array}$ & 4 & $X$ & & & Inglês \\
\hline $\begin{array}{c}\text { Latin American } \\
\text { Research Review }\end{array}$ & 3 & $\mathrm{X}$ & & & Inglês \\
\hline $\begin{array}{c}\text { Revista } \\
\text { Latinoamericana de } \\
\text { Politica Comparada }\end{array}$ & 2 & $X$ & $\mathrm{X}$ & $\mathrm{X}$ & Espanhol \\
\hline
\end{tabular}

Fonte: Elaboração dos autores.

Analisando as revistas na área de Ciência Política, uma revisão sumária do conteúdo das duas primeiras revistas citadas, especializadas em estudos comparativos, mostra uma presença bastante mais significativa de artigos focados na América Latina na revista Comparative Politics em relação à Comparative Political Studies. A presença de artigos focando a região também é presença constante no Journal of Democracy e na Revista de Ciencia Política, do Chile.

Porém, analisando o conteúdo dos textos, a referência à América Latina no título do artigo demonstrou não ser um indicador confiável para definir estados de natureza comparativa regional. Em grande parte dos casos estes se enquadravam na terceira categoria citada. A referência é feita à América Latina como um signo identificador para o leitor externo, em geral seguido da especificação "o caso do país X”, seguindo-se uma análise única de um caso nacional.

Mesmo na Revista Latinoamericana de Política Comparada, cujo nome identifica um foco duplo na comparação e no continente, embora efetivamente o espaço dado aos padrões de comparação do continente identificados nas categorias a e b - comparação extensiva e comparação de casos -, muitos dos textos usam a expressão conforme a categoria c, não se tratando de estudos comparativos continentais, mas estudos de caso nacionais, sendo latinoamericanos apenas pelo pertencimento à região, não havendo o desenvolvimento de categorias analíticas que possibilitem comparações empíricas.

No caso da Revista de Ciencia Política, do Chile, o número de artigos por número da revista é significativamente maior que o das demais, e, além disso, 
artigos envolvendo estudos de caso de países da América Latina têm dominado os números dos últimos anos. Este também é o caso de América Latina Hoy, em que alguns números são dedicados a dossiês de um único país, no entanto mesmo os números com dossiês temáticos, são constituídos majoritariamente pela análise de casos nacionais.

As diferenças de número de artigos publicados segundo a região do mundo em que focam podem ser atribuídas a vários fatores, como o tamanho da comunidade acadêmica e a língua de publicação. Embora um estudo mais aprofundado das origens e afiliações institucionais fosse necessário para corroborar a conclusão preliminar, há um indicativo de que grande parte dos artigos produzidos sobre a região é escrita por pesquisadores oriundos de fora da região.

Fazendo uma análise mais específica do caso brasileiro, a seguir se discute como o tema da política comparada e do continente tem sido tratado na ciênica política brasileira no período recente.

\section{0 que se publica no Brasil}

Uma das formas de observar o espaço dado para a política comparada na produção acadêmica brasileira é verificar quantos artigos vêm sendo publicados nas revistas científicas da área.

Foi analisada a produção de seis revistas brasileiras da área de Ciência Política, cinco delas com avaliação A, segundo o sistema Qualis da CAPES: Dados, publicada pelo Instituto de Estudos Sociais e Políticos (IESP); Opinião Pública, do Centro de Estudos sobre Opinião Pública da Universidade de Campinas (Cesop/Unicamp); Brazilian Polítical Science Review (BPSR), da Associação Brasileira de Ciência Política (ABCP); Revista Brasileira de Ciências Sociais (RBCS), da Associação Nacional de Pós-Graduação em Ciências Sociais (ANPOCS); Revista de Sociologia e Política (RSP), da Universidade Federal do Paraná (UFPR) e Revista Debates, do Núcleo de Pesquisas sobre a América Latina do Programa de Pós-Graduação em Ciência Política da Universidade Federal do Rio Grande do Sul (NUPESAL/UFRGS). Foram analisados os dados dos números do período 2006 a $2013^{2}$.

\footnotetext{
${ }^{2}$ Excluindo as edições do segundo semestre de 2013, ainda no prelo, em novembro. O estudo, originalmente preparado para uma mesa do VII Encontro da $\mathrm{ABCP}$, reunia dados até o ano de 2011.
} 
Quadro 2 - Artigos de política comparada em revistas brasileiras de ciência política 2006-2013

\begin{tabular}{|c|c|c|c|c|c|}
\hline Revista & $\begin{array}{c}\text { Números } \\
\text { por ano }\end{array}$ & $\begin{array}{c}\text { Número de } \\
\text { edições }\end{array}$ & $\begin{array}{c}\text { Total de } \\
\text { Artigos }\end{array}$ & $\begin{array}{c}\text { Artigos } \\
\text { Comparativos }\end{array}$ & $\%$ \\
\hline BPSR & 2 & 14 & 78 & $10(6)$ & 12,8 \\
\hline Dados & 4 & 31 & 217 & 13 & 6,0 \\
\hline Debates & 3 & 14 & 120 & 20 & 16,6 \\
\hline Opinião Pública & 2 & 15 & 131 & 12 & 9,1 \\
\hline RBCS & 3 & 23 & 239 & 8 & 3,3 \\
\hline RSP & 3 & 24 & 238 & 17 & 7,1 \\
\hline Total & - & 121 & 1023 & 103 & 10,0 \\
\hline
\end{tabular}

Fonte: Levantamento feito pelos autores.

As revistas têm diferentes perfis ${ }^{3}$. Algumas, como a RBCS e RSP publicam textos das áreas de ciências sociais em geral, e não exclusivamente da ciência política. A periodicidade varia entre revistas semestrais, quadrimestrais e trimestrais, e com tamanhos diferentes, o que explica a grande variação do número de artigos publicados.

Foram considerados os artigos que analisavam, de forma comparativa, dois ou mais países, independente da proposição teórica subjacente (análise econômica, de cultura ou de instituições políticas).

$\mathrm{O}$ que pode se verificar é uma presença relativamente pequena de artigos de caráter comparativo, perfazendo $10 \%$ do total de artigos publicados pelas revistas no período. A Revista Debates, publicada inicialmente por um Núcleo de Pesquisas dedicado ao continente, concentra o maior número de artigos comparativos em seus primeiros anos, rareando o tema no período mais recente, com exceção de um dossiê em 2013. A segunda revista com o maior percentual, a BPSR, com 12,8\% de artigos de caráter comparativo, apresenta somente seis de dez artigos publicados em um único número, dedicado a um dossiê sobre a América Latina.

Estes números tratam de artigos de natureza comparativa. Em muitos casos, as comparações incluem o Brasil mas não outros países da América Latina.

O maior espaço das revistas é ocupado por textos sobre a política nacional, seguidos de textos de análise teórica. As contribuições, quando referentes a outros países, por autores estrangeiros ou nacionais, costumam ser igualmente de estudo de caso individual.

Esta é, obviamente, uma observação parcial. Estas revistas representam uma parcela pequena dos textos de ciência política publicados no Brasil. Os autores que se dedicam à comparação podem estar publicando em outras partes. Porém esta amostra é um indicativo da orientação da elite intelectual da área, que edita as revistas e nelas publica.

\footnotetext{
${ }^{3}$ Análise mais aprofundada do conteúdo destas revistas pode ser encontrada em Soares (2005) e Valle Silva (1999).
} 
Como nem todas as revistas publicam a filiação institucional dos autores, torna-se difícil fazer um mapeamento confiável das origens intelectuais e profissionais dos mesmos. Uma observação geral indica que as revistas reproduzem a situação da área de ciência política no Brasil, com um domínio de Rio de Janeiro e São Paulo, com uma presença relativamente forte de Rio Grande do Sul e Minas Gerais, um pouco menos marcante de Paraná, Pernambuco e Brasília, sendo mais rara e episódica a presença fora deste circuito. A presença de autores estrangeiros indica um predomínio de estadunidenses, argentinos, uruguaios e chilenos, com aparições esporádicas de autores de outros países.

Não se observa o crescimento do número de artigos comparativos ao longo dos anos, indicando que uma possível expansão da ciência política no Brasil, em relação ao cenário descrito por Amorim Neto e Santos (2005), não se deu de forma particular no âmbito dos estudos comparados ou com enfoque na América Latina. Igualmente a consolidação da ALACIP (Associação Latino-americana de Ciência Política), com a realização de congressos periódicos, inclusive no Brasil, não parece ser estímulo suficiente para o surgimento de novos comparativistas.

Como agravante há a tendência, que pode ser verificada no padrão da lista Qualis da ciência política, com pequeno número de revistas publicadas na América Latina, de considerar Europa e Estados Unidos como único modelo acadêmico, relegando nossa produção a um papel periférico.

\section{Como se ensina a política comparada no Brasil}

Em primeiro lugar, o estudo da ciência política é afetado por uma diferença entre o Brasil e diversos outros países do continente, em termos de organização de carreiras acadêmicas: a quase inexistência de cursos de formação específica em ciência política. A formação de cientistas políticos como carreira profissional, com atuação fora da academia, é um processo recente e embrionário no Brasil. Nos cursos de graduação a ciência política em geral é uma das áreas de formação dos cursos de ciências sociais, junto à sociologia e à antropologia. São poucos e de criação recente os cursos específicos de graduação em Ciência Política, exceção feita à Universidade de Brasília. A especificidade da área em geral era adquirida por meio da Pós-Graduação, nos mestrados e doutorados, voltados em geral para a formação de professores e pesquisadores ${ }^{4}$.

O ingresso aos cursos de pós-graduação não é, em geral, restrito aos graduados em ciências sociais, sendo comum o ingresso de oriundos das carreiras de direito, economia, história, filosofia e mesmo de áreas menos afins, como jornalismo ou engenharia, em geral com formação incipiente em métodos de pesquisa social, transferindo para a pós-graduação a responsabilidade pelo preenchimento desta lacuna.

\footnotetext{
${ }^{4}$ Sobre a trajetória da Ciência Política no Brasil ver Amorim e Santos (2005).
} 
Para verificar a importância dada ao campo da política comparada no processo de formação, analisou-se os currículos dos oito cursos de pós-graduação em Ciência Política com notas entre 5 e 7, segundo a CAPES (Universidade Nacional de Brasília, Universidade Federal de Minas Gerais, Universidade de São Paulo, Instituto Universitário de Pesquisas do Rio de Janeiro, Instituto de Estudos Sociais e Políticos, Universidade Federal de Pernambuco, Universidade Estadual de Campinas e Universidade Federal do Rio Grande do Sul). Além de serem considerados os centros de excelência, são os cursos que teoricamente tem inserção internacional, o que, em tese, favoreceria uma perspectiva comparativa. Analisaram-se também os currículos de graduação em ciências sociais das mesmas instituições. Todos os cursos analisados possuem uma linha de pesquisa ou um centro de estudos que inclua o tema da comparação política entre seus objetivos.

Nos currículos de graduação, em geral, está presente uma disciplina de política comparada, porém como eletiva, isto é, o aluno não é obrigado a cursá-la podendo optar por outras. No caso da pós-graduação está presente em todos os cursos o tema da política comparada, diferindo a forma como é abordado: como disciplina específica ou como parte dos conteúdos de metodologia. Em alguns cursos é obrigatório, em outros, eletivo.

A tentativa de levantar o número de teses e dissertações recentes com tema comparativo esbarrou na ausência de uma padronização no uso de palavras-chave e na dificuldade de detectar, pelos títulos, a natureza comparativa dos trabalhos.

Para um período anterior, Maria Helena Santos e Marcelo Coutinho analisaram 955 teses defendidas entre 1985 e 2000, buscando estudos comparados. Concluíram que "nos dez centros de pós-graduação da área da CAPES de ciência política, entendendo-se por comparados os estudos que analisam mais de dois países, somente 3\% usavam essa perspectiva e, entre eles, nenhum usava dados quantitativos" (SANTOS e COUTINHO apud SOARES 2005, p. 28).

Conclui-se que a formação na metodologia comparativa está disponível, mas nem sempre é considerada essencial, sendo possível haver egressos que não tenham tido contato com este campo em toda a sua formação acadêmica. Isto talvez explique tanto os dados de Santos e Coutinho como os resultados apresentados na análise das revistas.

\section{Por que se resiste ao método comparativo empírico?}

De maneira geral, os métodos quantitativos utilizados na política comparada têm sido historicamente vistos com desconfiança pelos cientistas sociais na América Latina.

Pelos dados apresentados neste texto em relação ao uso de metodologia quantitativa na política comparada se constata que o debate metodológico na Ciência Política ocorre num plano secundário, o que contrasta com a carência na 
formação de recursos humanos nesta área. Igualmente se verifica que os recursos humanos formados no campo da Ciência Política, na sua maioria, apresentam lacunas no que diz respeito a como fazer pesquisa comparativa e, sobretudo, em relação à compreensão do processo de desenvolvimento de uma pesquisa comparativa, na dimensão quantitativa.

No âmago da divergência a respeito da utilidade, ou não, de métodos quantitativos comparativos, subjaz a ideia de que fenômenos nesta área são mais bem explicados por teorias normativas do que por descrições consideradas pseudo-precisas.

Neste texto parte-se do pressuposto que o profissional da área de Ciência Política necessita dominar as diferentes técnicas da metodologia de pesquisa, pois ele não é só um "consumidor" da pesquisa, mas dele se espera também contribuições no processo de construção de conhecimento. Toda e qualquer técnica de análise tem limites e deficiências. No entanto, a necessidade de compreender com mais profundidade as diferenças e semelhanças entre os países da América Latina compele o pesquisador a conhecer as possibilidades e limites dos diferentes métodos para construir um conhecimento contextualizado sobre a realidade dessas sociedades.

Por essa, entre outras razões, pensa-se que os cursos de metodologia comparativa estão em crise. Por um lado, os alunos se sentem prejudicados por não terem oportunidades de "praticarem" diferentes tipos de pesquisa. No caso do Brasil, de acordo com Schwartzman, "academicamente, se avançou muito nas publicações, mas não tanto do ponto de vista das aplicações de uso da ciência" (SCHWARTZMAN, 2007, p. 1).

Contemporaneamente, parece haver um consenso de que a formação no campo da Ciência Política exige o domínio de uma gama de diferentes métodos de pesquisa. Segundo Hentschel (2002), a redução da investigação em política comparada a um único método de pesquisa denominado de quali-quantitativo está superada pela necessidade de conhecer e utilizar o que se tem denominado de pesquisa quali-quantitativa. Tais esforços parecem ser urgentes no campo da política comparada. No entanto, alguns autores têm apontado que existe, atualmente, um déficit de cientistas políticos com competência suficiente para desenvolver estudos quantitativos (LEWIS, 2003).

No contexto atual do conhecimento da Ciência Política, no qual cada vez mais se torna imprescindível gerar novas bases de conhecimento, em virtude da defasagem teórico-explicativa de fenômenos sociais contemporâneos, é necessário romper com princípios que têm se mantido ao longo do tempo. A adesão a uma única forma de construir conhecimento sem questionar suas implicações teóricas, econômicas e sociais, pode redundar no estabelecimento do conhecimento reativo, ou seja, há uma tendência a reagir o que vem de fora do que a criação de categorias contextualizadas ao cenário que se está estudando. E isso, em 
detrimento da construção de um conhecimento mais concreto da realidade na qual estamos inseridos.

Desde que os cientistas políticos passaram a ter espaço próprio, desenvolveram teorias e métodos para ajudá-los a compreender o comportamento social e político das pessoas e de sistemas políticos. Nessa perspectiva, Sandres (1976) compara detetives a cientistas sociais no sentido de que os dois formulam teorias e desenvolvem métodos na tentativa de responder a duas questões: "por que aconteceu" e "em que circunstâncias provavelmente irão acontecer novamente". Desse modo, a pesquisa quantitativa tem como objetivo principal explicar e predizer. Nesse tipo de pesquisa, os cientistas políticos, para testar suas teorias, dependem de evidência empírica e de modalidades lógicas de análise. Utilizam técnicas de observação, entrevistas, experimentos e outros métodos empíricos para testar a validade dos seus resultados.

Entretanto, nos nossos países tem se materializado o conhecimento normativo e reativo, desestimulando o processo de construção de conhecimento novo e autóctone com base em determinadas condições culturais e sociais. Tal conhecimento se torna necessário para preencher os déficits explicativos da maior parte dos temas contemporâneos.

Não é incomum a Ciência Política se defrontar com resultados, em estudos sobre a mesma temática, utilizando ou técnicas quantitativas ou qualitativas, com resultados que frequentemente se contradizem. De um lado, tal processo é normal, pois a polêmica é parte essencial da pesquisa. Porém, por outro lado, tais contradições ocorrem pelo uso inapropriado de técnicas de análise e de estruturação de projetos. Essa situação decorre, às vezes, de posturas distorcidas de pesquisadores que sugerem e defendem o uso de um único método, mesmo quando a natureza dos fenômenos sociais sugere outros caminhos de análise. $\mathrm{O}$ bom e competente pesquisador domina plenamente as várias técnicas de pesquisa sem confundir epistemologia com técnica.

Ao lado disso, como observa o mesmo autor, a crise das Ciências Sociais se agrava, entre outros motivos, pela posição cada vez mais frequente dos pesquisadores latino-americanos de assumirem uma postura de submissão ao conhecimento estabelecido que domina a área. Dietrich é contundente ao afirmar que:

Enquanto o regime garante os privilégios dos intelectuais acadêmicos, estes se abstêm de gerar teorias críticas e formar alunos críticos, que poderiam perturbar o status quo. Aceitam uma existência de banalidade enquanto o regime aceita a ficção de que se realiza Ciência Social nas academias. Tal trato é unidirecional. A única saída para deixar a banalidade para trás é ir adiante, em direção à criação de novos mitos de dominação a serviço das elites. Dessa forma, o professor se converte em um padre secular, cujo desempenho se esgota nos cânones e liturgias da teologia política do sistema (DIETRICH, 2002, p. 13). 
De maneira geral, adaptar-se, no contexto latino-americano, tem significado aceitar passivamente a destruição de valores culturais próprios e a internalização de hábitos e comportamentos que nada têm a ver com o sistema do qual fazemos parte. $\mathrm{O}$ resultado desse processo tem-se plasmado em teorias vigentes que não explicam adequadamente os fenômenos sociais, adotando como base um pensamento diferente.

Uma consequência deste processo, segundo Gomes (1998, p. 2), é de que “os 'pesquisadores' têm se debruçado sobre questões de relevância duvidosa, perdendo-se nos meandros das obrigações burocráticas da academia, sem efetivamente conseguir produzir algo de novo", e continua: "e se não bastasse essa limitação, temos por outro lado um enorme desperdício de tempo e dinheiro na produção de estudos irrelevantes, geralmente condenados ao ostracismo do fundo de nossas bibliotecas".

\section{0 que fazer?}

Neste caso a solução provavelmente não está somente em um grupo pequeno e altamente especializado de profissionais, embora também depende deles, já que a ciência política que fazemos hoje dificilmente atinge as massas, nem ao menos as acadêmicas.

Uma iniciativa que possibilitou a ampliação do volume de recursos e editais para fomentar a cooperação no Cone Sul, aprovada desde 2004, foi a implementação, em 2011, do Fundo Educacional do MERCOSUL.

As

facilidades de uso da Internet necessitariam ser potencializadas, com a realização de eventos e debates por videoconferência e o compartilhamento de publicações e dados em formato eletrônico.

A disponiblidade de dados de pesquisas comparativas de consórcios internacionais aumentou muito nos últimos anos, podendo ser destacados os dados do Latinobarômetro, do World Values Survey, do Barômetro das Américas (LAPOP), entre outros.

Uma cooperação de longo prazo, entre pesquisadores da América Latina, passa pela valorização do estudo da língua dos países vizinhos - o uso do português e do espanhol propriamente ditos, e não conformar-se com um, às vezes, constrangedor "portunhol".

Os estudantes deveriam ser estimulados a estudar e produzir trabalhos que incorporem informação sobre os países da região. Neste sentido, na qualidade de professores, avalia-se como positiva a experiência com alunos de política comparada na graduação de ciências sociais e relações internacionais, realizando com os alunos atividades práticas de aplicação do método comparativo, demonstrando que seu uso pode ser importante não apenas no mundo teórico abstrato, mas também na análise política e na tomada de decisões. $\mathrm{O}$ ideal seria 
que fosse obrigatório para todo candidato a doutor em ciência política cursar disciplinas de política comparada.

Igualmente, é preciso ampliar o intercâmbio de alunos e pesquisadores, possibilitando o contato com bibliografia e realidades mútuas. Este processo passa também pela incorporação, nas atividades docentes, de bibliografia produzida nos países vizinhos, o que exige um esforço de melhoria de distribuição desse material, sendo hoje mais fácil adquirir um livro publicado nos EUA do que em Montevidéu, Buenos Aires ou Santiago.

Este esforço passa também por uma descolonização intelectual, deixando de ver o mundo acadêmico do hemisfério norte como o único padrão a ser seguido e a única fonte de teorias e métodos. Em síntese, sem renegar as diversas influências epistemológicas na política comparada, é necessário valorizar a produção teórica e metodológica local.

Rodrigo Stumpf González é Professor do Programa de Pós-Graduação em Ciência Política da Universidade Federal do Rio Grande do Sul (UFRGS). E-mail: rsg65@terra.com.br

Marcello Baquero é Professor do Programa de Pós-Graduação em Ciência Política da Universidade Federal do Rio Grande do Sul (UFRGS). E-mail: nupesal@ufrgs.br

\section{Referências}

AMORIM NETO, Octavio; SANTOS, Fabiano. La Ciencia Política en el Brasil: El Desafío de la Expansión. Revista de Ciencia Política, v. 25, n. 1, p. 101-110, 2005.

DAHL, Robert A. Political oppositions in Western Democracies. New Haven: Yale University Press, 1966.

DIETRICH, Heins. Identidade nacional e globalização: a Terceira via - Crise nas Ciências Sociais. Blumenau: Edifurb, 2002.

DURKHEIM, Emile. As regras do método sociológico. São Paulo: Companhia Editora Nacional, 1987.

GOMES, Alberto Albuquerque. Considerações sobre a pesquisa científica: Em busca de caminhos para a pesquisa científica. 1998. Disponível em: <http://www.fct.unesp.br/Home/Departamentos/Educacao/AlbertoGomes/aula _consideracoes-sobre-a-pesquisa.pdf>. Acesso em: 28 de outubro de 2013.

HENTSCHEL, Hartmut. Ecuestas y opinion publica: aspectos metodologicos. Buenos Aires: Edivérn, 2002. 
HUNTINGTON, Samuel P. Political order in changing societies. New Haven: Yale University Press, 1968.

MACRIDIS, Roy; BROWN, Bernard. Comparative Politics - notes and readings. Homewood: Dorsey Press, 1968.

LANE, Ruth. The Politics model: Political Science in theory and practice. New York: M. E. Sharpe, 1997.

LEWIS, Jane. How useful are Social Sciences? Political Quarterly, v. 74, n.2, p. 193-201, 2003.

MIGDAL, Joel S. Strong societies and weak states: State-society relations and state capabilities in the third world. Princeton: Princeton University Press, 1988.

MOORE, Barrington. The social origins of dictatorship and democracies. Boston: Beacon Press, 1966.

NORTH, Douglass C.; THOMAS, Robert P. The rise of the western world. A new economic history. Cambridge: Cambridge University Press, 1973.

PRZEWORSK, Adam; TEUNE, Henry. The logic of comparative social inquiry. Michigan: Wiley-Interscience, 1970.

PRZEWORSKI, Adam; SPRAGUE, John. Paper Stones: A history of electoral socialism. Chicago: University of Chicago Press, 1986.

SANDERS, Willian B. Sociologist as detective: an introduction to research methods. New York: Praeger Publishers, 1976.

SOARES, Gláucio Ary Dillon. O calcanhar metodológico da ciência política no Brasil. Sociologia, Oeiras, n. 48, p. 27-52, maio, 2005.

SCHWARTZMAN, Simon. O crítico da Ciência. Pesquisa Fapesp. Edição impressa. São Paulo, n. 140, out. 2007. Entrevista concedida a Carlos Fioravanti. 2007.

VALLE SILVA, Nelson. Relatório de Consultoria sobre Melhoria do Treinamento em Ciência Social Quantitativa e Aplicada no Brasil. Rio de Janeiro: Laboratório Nacional de Computação Científica, 1999.

\section{Revistas analisadas}

AMÉRICA LATINA HOY. Disponível em: <http://campus.usal.es/ revistas_trabajo/index.php/1130-2887/index>.

BRAZILIAN POLITICAL SCIENCE REVIEW. Disponível em: $<$ http://www.bpsr.org.br/index.php/bpsr/index>.

COMPARATIVE POLITICS. Disponível em: $<$ http://web.gc.cuny.edu/jcp/index.HTM>.

DADOS - REVISTA DE CIÊNCIAS SOCIAIS. Disponível em: <http://www.scielo.br/revistas/dados/iaboutj.htm>.

JOURNAL OF DEMOCRACY. Disponível em: $<$ http://www.journalofdemocracy.org/>. 
LATIN AMERICAN POLITICS AND SOCIETY. Disponível em: <http://onlinelibrary.wiley.com/journal/10.1111/\%28ISSN\%2915482456/issues>.

LATIN AMERICAN RESEARCH REVIEW. Disponível em: $<$ http://lasa.international.pitt.edu/eng/larr/index.asp>.

OPINIÃO PUBLICA.

Disponível

em: <http://www.scielo.br/revistas/op/iaboutj.htm>.

REVISTA LATINOAMERICANA DE POLÍTICA COMPARADA. Disponível em: <http://www.politicacomparada.com/revista.html>.

REVISTA DE CIÊNCIA POLÍTICA. Disponível em:

$<$ http://www.scielo.cl/scielo.php?script=sci_serial\&pid=0718-090X >.

REVISTA DEBATES.

Disponível

em: <http://seer.ufrgs.br/debates/issue/archive>.

REVISTA DE SOCIOLOGIA E POLÍTICA. Disponível em: $<$ http://www.scielo.br/revistas/rsocp/iaboutj.htm>.

Texto recebido em 12 de novembro 2013. Aprovado em 02 de dezembro de 2013. 